\title{
ARTICLE Goblet cell associated antigen passages support the induction and maintenance of oral tolerance
}

\author{
Devesha H. Kulkarni ${ }^{1}$, Jenny K. Gustafsson ${ }^{1}$, Kathryn A. Knoop ${ }^{1}$, Keely G. McDonald ${ }^{1}$, Shay S. Bidani ${ }^{1}$, Jazmyne E. Davis ${ }^{1}$,
} Alexandria N. Floyd ${ }^{1}$, Simon P. Hogan ${ }^{2}$, Chyi-Song Hsieh ${ }^{1}$ and Rodney D. Newberry ${ }^{1}$

Tolerance to innocuous antigens from the diet and the commensal microbiota is a fundamental process essential to health. Why tolerance is efficiently induced to substances arising from the hostile environment of the gut lumen is incompletely understood but may be related to how these antigens are encountered by the immune system. We observed that goblet cell associated antigen passages (GAPs), but not other pathways of luminal antigen capture, correlated with the acquisition of luminal substances by lamina propria (LP) antigen presenting cells (APCs) and with the sites of tolerance induction to luminal antigens. Strikingly this role extended beyond antigen delivery. The GAP function of goblet cells facilitated maintenance of pre-existing LP T regulatory cells (Tregs), imprinting LP-dendritic cells with tolerogenic properties, and facilitating LP macrophages to produce the immunomodulatory cytokine IL-10. Moreover, tolerance to dietary antigen was impaired in the absence of GAPs. Thus, by delivering luminal antigens, maintaining pre-existing LP Tregs, and imprinting tolerogenic properties on LP-APCs GAPs support tolerance to substances encountered in the hostile environment of the gut lumen.

Mucosal Immunology (2020) 13:271-282; https://doi.org/10.1038/s41385-019-0240-7

\section{INTRODUCTION}

The single layer epithelium lining the gastrointestinal (Gl) tract is the interface between the host and the luminal environment containing trillions of microbes. At this site, the immune system encounters an array of foreign substances, ranging from innocuous dietary antigens and commensal microbes to pathogens. Responding appropriately to each of these is critical to maintaining immune homeostasis in this potentially hostile environment. Paradoxical to the hostile environment of the gut lumen, steady state encounters with non-pathogenic antigens originating from this site result in the induction of antigen specific tolerance, which is largely mediated by CD4+ Foxp3+ T regulatory cells (Tregs). ${ }^{1}$ Despite advances in our understanding of mechanisms inducing antigen specific Tregs, why tolerance is so efficiently induced to antigens originating from this particularly hostile environment of the gut lumen remains incompletely understood.

The intestinal lamina propria (LP) contains an array of antigen presenting cells (APCs), including classical CD103+CD11b- IRF8 dependent dendritic cells (DCs), IFR4 dependent CD103+CD11b + DCs, and CD103-CD11b+ cells that can express IRF4 and can include resident macrophages, with the $C D 103+C D 11 b+$ and CD103-CD11b + APCs making of the majority of the population in the LP. ${ }^{2-7}$ Collectively these cellular populations, excluding $B$ lymphocytes, will be referred to as LP-APCs. While each subset preferentially supports various phenotypes of antigen specific $T$ cell responses, there is an evolving understanding that they may play redundant roles in the induction of oral tolerance. ${ }^{8}$ While tolerogenic responses can be induced in Peyer's Patches and potentially in other mucosal lymphoid tissues, it has become appreciated that the gut draining lymph nodes are critical sites for the induction of oral tolerance., ${ }^{9} 10$ Current understanding is that this process requires the acquisition of antigens by LP-APCs underlying the villous epithelium, their trafficking to the draining lymph nodes to induce naive CD4 $+T$ cells to differentiate into peripherally induced Tregs (pTregs), and homing of these pTregs to the LP where they are maintained by continued stimulation by LP-APCs acquiring the cognate antigen for these pTregs from the lumen. ${ }^{11,12}$ Tolerance to luminal antigens occurs in the small intestine $(\mathrm{SI})^{13}$ and in the distal colon, ${ }^{14}$ indicating these are the sites where luminal antigens cross the epithelium and are acquired by LP-APCs. How antigens are captured by LP-APCs at these sites may be the basis for why tolerance is effectively induced in this hostile setting.

Several routes by which luminal substances cross the epithelium have been identified including paracellular leak, the direct capture by LP-APCs via extension of trans-epithelial dendrites (TEDs) into the gut lumen, passage from the lumen via villous $M$ cells, and passage from the lumen via goblet cell associated antigen passages (GAPs). ${ }^{15-22}$ Of these, LP-APC extension of TEDs is the currently favored route to support the induction and maintenance of tolerance to luminal substances in the steady state, as the extension of TEDs does not compromise the epithelial barrier and would allow direct acquisition of luminal antigens by LP-APCs. ${ }^{16}$ However, this process directly exposes the LP-APCs to luminal contents, which in vitro studies indicate induces mixed Th1 and Th2 responses. ${ }^{23}$ In addition, TEDs are absent in some mouse strains, which do not display defects in oral tolerance ${ }^{24}$ and are lacking in regions of the gut where gavaged antigen is

\footnotetext{
${ }^{1}$ Department of Internal Medicine, Washington University School of Medicine, Saint Louis, MO 63110, USA and ${ }^{2}$ Mary H. Weiser Food Allergy Center, University of Michigan School of Medicine, Ann Arbor, MI 48109, USA

Correspondence: Rodney D. Newberry (rnewberry@wustl.edu)

These authors contributed equally: Devesha H. Kulkarni, Jenny K. Gustafsson
}

Received: 7 February 2019 Revised: 31 October 2019 Accepted: 18 November 2019

Published online: 9 December 2019 
captured by LP-APCs ${ }^{25,26}$ suggesting that other luminal antigen acquisition pathways could support oral tolerance. Thus, how luminal antigens are acquired by LP-APCs for the induction of tolerance and if this process is integral to efficiently inducing tolerance in the hostile gut luminal environment remain unclear. Here we evaluated steady state routes of luminal antigen capture by LP-APCs. We found that LP-APC extension of TEDs, villous M cells and paracellular leak did not correlate with effective antigen capture by LP-APCs. In contrast the density of GAPs directly correlated with LP-APC luminal antigen capture and with the regions within the gut where tolerance is induced to luminal substances. Moreover, beyond the role of antigen delivery, we find that the GAP function of goblet cells imprints and maintains LPDCs and macrophages with tolerogenic properties, maintains preexisting Tregs in the SI LP, and in the absence of GAPs tolerance to dietary antigens is impaired. Thus, the GAP function of goblet cells acts as both a pathway to deliver luminal substances to LP-APCs and as a mechanism imprinting LP-APCs with tolerogenic properties to maintain and induce tolerance to antigens encountered in the hostile environment of the gut lumen.

\section{RESULTS}

The presence of goblet cell associated antigen passages (GAPs), but not LP-APC extension of TEDs or villous M cells, correlates with the sites of luminal antigen capture for the induction of tolerance In the steady state, tolerance to luminal substances is induced in the $\mathrm{SI}$ and distal colon. ${ }^{13,14}$ How luminal substances cross the epithelium to be encountered by the immune system is a fundamental process that may underlie why tolerance is so efficiently induced to substances arising from an unfavorable environment with abundant microbes and microbial products. To evaluate how dietary antigen traverses the intestinal epithelium we performed intraluminal injections of fluorescently labeled ovalbumin (Ova) and evaluated fixed intestinal sections by fluorescent microscopy. Immunofluorescent staining of fixed tissue sections demonstrated that goblet cells containing the luminally administered fluorescent Ova could be identified throughout the $\mathrm{SI}$ and in the distal descending colon and sigmoid colon, referred to as the distal colon, but were less common in the cecum, ascending colon, transverse colon, and proximal descending colon, referred to as the proximal colon (Fig. 1a-d). The presence of GAPs in the distal colon was not appreciated in the work initially identifying GAPs using the in vivo imaging approach due to the difficulty of imaging the distal colon with this approach. This regional distribution of GAPs correlates with the previously identified lymph nodes draining the regions of the gut supporting tolerance. $^{13,14}$ Secretory intestinal epithelial cell lineages other than goblet cells have been observed to take up luminal antigens. $^{27,28}$ We observed that Paneth cells containing luminally administered Ova were present throughout the length of the SI but significantly less common when compared to goblet cells containing fluorescent Ova (Fig. 1a, b). We identified a small number of enteroendocrine cells containing luminally administered Ova in the steady state that were restricted to the duodenum; these were also significantly less common than goblet cells containing fluorescent Ova (Fig. 1a, b). In addition, we did not observe $M$ cells in the non-follicle bearing epithelium in the SI or colon in the steady state (Fig. S1).

The currently favored route of luminal antigen acquisition by LP-APCs for tolerance induction is direct capture through the extension of TEDs. We evaluated the frequency and regional distribution of TED extension by LP-APCS by in vivo two-photon imaging of $C D 11 c^{\text {YFP }}$ and $C X_{3} C R 1^{\text {GFP }}$ reporter mice. Mice were imaged at various times throughout the day and were not deprived of food or water prior to imaging. At steady state conditions, we observed LP-APC extension of TEDs to be very rare in the distal SI and absent in the proximal SI (Fig. S1 B-E).
We observed two TEDs that were located in the distal SI out of greater than 500 villi imaged from tip to base from multiple CD11 $c^{\text {YFP }}$ reporter mice (Fig. S1D left side) and four TEDs forming in the distal SI out of greater than 350 villi imaged from tip to base throughout the $S I$ from $C_{3} C R 1^{G F P / W T}$ reporter mice (Fig. S1E left side). We did not observe any TED extension in either the proximal or distal colon after analyzing 260 colonic crypts in the CD11c ${ }^{\text {YFP }}$ reporter mice and 263 crypts in the $C_{3} C R 1^{G F P}$ reporter mice (Fig. S1D and E, left side).

Previous studies removing the luminal contents mucus by washing, identified TED extension by APCs occurred at a rate of 1.5-2.0 TEDs/villus. ${ }^{15,24,25,29,30}$ Approximately ten minutes following the removal of the luminal contents and mucus by rinsing with PBS, LP-APCs became less compact and extended multiple dendrites within the LP, into the epithelium, and into the lumen, with some LP-APCs traversing the epithelium (Supplemental Movie S1). However, consistent with prior observations, ${ }^{15,25}$ $\mathrm{CX}_{3} \mathrm{CR} 1^{\mathrm{GFP}+}$ LP-APC TED formation did not occur in the duodenum, the site where gavage antigen is acquired by $\mathrm{CX}_{3} \mathrm{CR}^{\mathrm{GFP}+}$ LP-APCs ${ }^{26}$ (Fig. S1E right side), and TED formation was not observed in the distal colon in any condition (Fig. S1B-E), consistent with observations by others that TEDs are rare or absent in the colon. ${ }^{31,32}$

Using the in vivo two-photon imaging approach we used to evaluate the frequency of TEDs, we evaluated the frequency of GAPs in villi and colonic crypts. We did not observe an effect of removal of the mucus layer on the frequency of GAPs and the regional distribution of GAPs remained similar to our findings of GAPs using fluorescent microscopy on fixed tissue sections (data not shown). In the SI GAPs were 1000 fold more common than TEDs when the mucus layer was left intact and $\sim 10$ fold more common when the mucus layer was removed (Fig. 1E). Thus, the frequency and regional distribution of GAPs, but not luminal antigen acquisition by Paneth cells, or enteroendocrine cells, the presence M cells, or LP-APC extension of TEDs correlated with regions of the gut where tolerance to luminal substances can be induced. ${ }^{13,14}$

GAPs support LP-APC capture of, and CD4+ T cell responses to, luminal antigen

Mouse atonal homologue 1 (Math1) is a transcription factor required for the development of neurons and intestinal secretory intestinal epithelial lineages, which includes goblet cells, enteroendocrine cells, and Paneth cells. ${ }^{33-36}$ Paneth cells have a significantly longer half-life than goblet cells, ${ }^{37,38}$ and accordingly, 10 days after treatment with tamoxifen, mice with an inducible deletion of Math1 in intestinal epithelial cell lineages (Math ${ }^{\mathrm{fl} / \mathrm{fl}} \mathrm{vil}$ Cre-ER ${ }^{\mathrm{T} 2}$ mice) lose goblet cells (Fig. S2A), but retain Paneth cells, albeit at a somewhat reduced number when compared to their littermate controls (Fig. S2B). Goblet cells acquiring luminal fluorescent dextran in the $\mathrm{SI}$ and distal colon decreased significantly 10 days following the deletion of Math 1 in intestinal epithelial cells (Fig. S2C and D). In contrast to the decrease in GAPs, intestinal permeability increased, as evidenced by serum levels of $4 \mathrm{kD}$ FITC dextran following gavage (Fig. 2a), and as evidence by the presence of $3 \mathrm{kD}$ fixable FITC dextran between epithelial cells and within the lamina propria of goblet cell deficient mice following gavage (Fig. S2E). The increased permeability might be attributed to the loss of the mucus barrier following goblet cell deletion. Despite the increase in intestinal permeability, SI LP-APCs, identified by flow cytometry (Fig. S3), acquired less luminally administered fluorescent Ova (Fig. 2b, c). In addition to the effects rising from the loss of GAPs, this may in part be related to the size of intact Ova $(\sim 43 \mathrm{kD})$, as gavage of $40 \mathrm{kD}$ FITC dextran did not result in increased serum levels in goblet cell deficient mice (Fig. 2a) and gavaged fluorescent Ova was not found leaking between SI epithelial cells or in the lamina propria of goblet cell deficient mice (Fig. S2E). Isolation of the CD103+ 
a

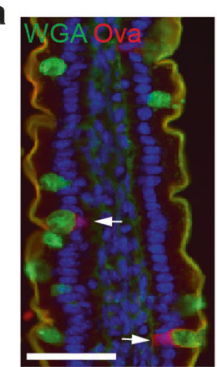

c

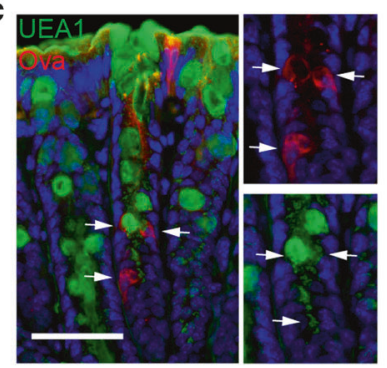

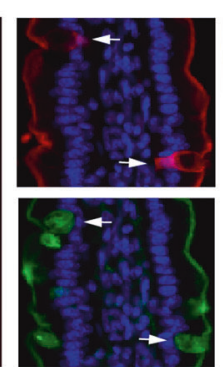
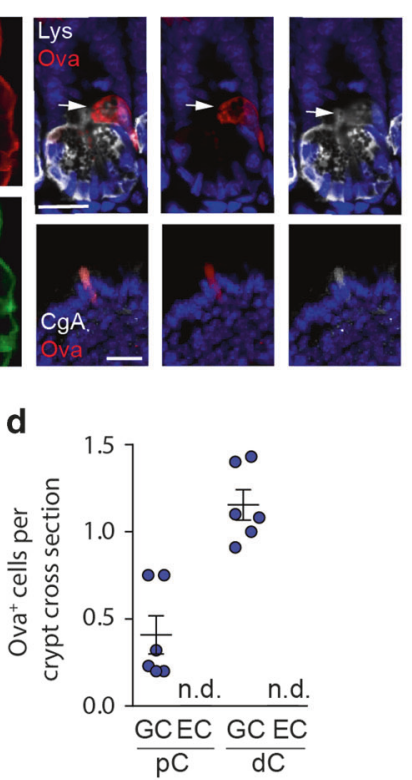

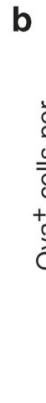

e
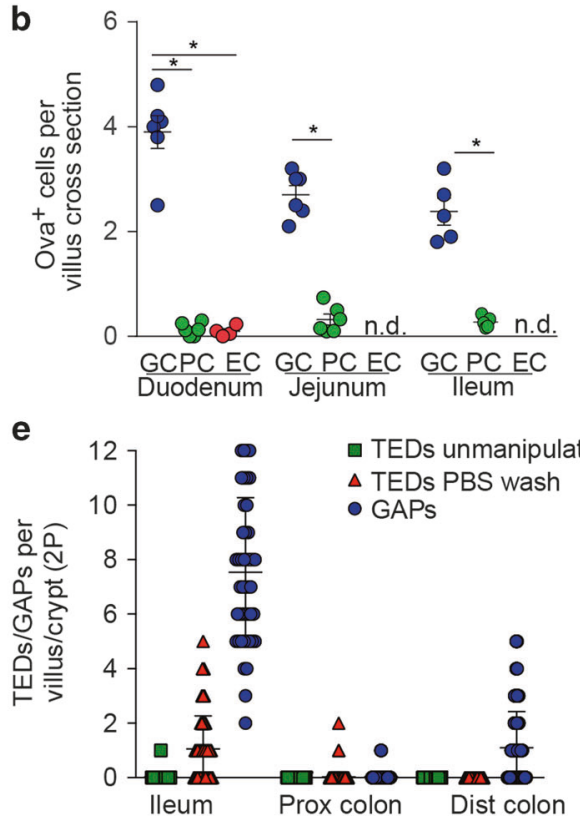

Fig. 1 Goblet cell associated antigen passages (GAPs) are present at the sites where tolerance to luminal substances is induced in the steady state. a representative images and b quantification of goblet cells (GC; wheat germ agglutinin (WGA)+ in SI), Paneth cells (PC; lysozyme (Lys) + ), and enteroendocrine cells (EC; chromogranin A (CgA)+ ) taking up luminal fluorescent ovalbumin (Ova) in regions of the SI determined by immunofluorescent staining on fixed tissue sections. c Representative images and $\mathbf{d}$ quantification and of goblet cells (Ulex Europaeus Agglutinin I (UEAI)+ in colon) and ECs taking up luminal fluorescent Ova in the proximal colon (pC) and distal colon (dC) as determined by immunofluorescent staining of fixed tissue sections. e Quantification of TEDs and GAPs per SI villus or colon crypt obtained via in vivo two-photon imaging. Scale bar $=50 \mu \mathrm{m}$ in large panels a and $\mathbf{c}$ and $20 \mu \mathrm{m}$ in small panels a, Each data point represents an individual mouse with 30 or more villi and 40 or more crypts evaluated per mouse in panels $\mathbf{b}$ and $\mathbf{d}$. Each data point in panel e represents an individual crypt or villus. Data are presented as the mean \pm SEM. ${ }^{*} P<0.05$, ns not significant, n.d. not detected.

CD11b+ APC population and the CD103-CD11b+ APC population, which may contain DCs and macrophages, following Ova gavage revealed that the APCs were no longer of inducing Ova specific CD4+ T cell responses in ex vivo co-cultures when goblet cells and GAPs were absent (Fig. 2d). We were unable to isolate sufficient numbers of CD103+CD11b-SI LP-DCs for this ex vivo assay. The impaired ability of LP-APCs to induce T cell proliferation to luminal antigen was not due to an intrinsic defect in antigen acquisition or presentation, as LP-APCs isolated from mice with Math1 deleted in intestinal epithelial cells displayed no defects in capture of fluorescent antigen in culture (Fig. S4A) and no defect in induction of $T$ cell proliferation when exogenous Ova was added to ex vivo co-cultures (Fig. S4B). We attributed the decrease in LP-APC antigen acquisition to the loss of goblet cells and GAPs, as we saw very few intestinal enteroendocrine cells and few Paneth cells acquiring luminal Ova in wild-type mice (Fig. 1a, b), and Paneth cells were still present at this time following deletion of Math1 (Fig. S2B). Moreover, mice lacking goblet cells/GAPs were significantly impaired at inducing antigen specific CD4+ $T$ cell responses to gavaged antigen in the SI draining MLN (Fig. 2e), the site of tolerance induction to dietary antigens. The impaired responses to luminal antigen were not attributable to defects in the ability of MLN T cells to respond to Ova, as responses to systemically administered Ova were not impaired (Fig. S4C). The CSFE dilution seen in the mice lacking goblet cells may be due to antigen acquired at other sites, such as the Peyer's Patches and migration of DCs to the MLN, as we saw no proliferation of OTII Rag-/- T cells, which have TCR specificity only for Ova, in the MLN in the absence of Ova gavage, and reduced but detectable proliferation of OTII Rag-/- T cells in the MLN in response to Ova in mice lacking goblet cells and GAPs when compared with their Cre- littermates (Fig. S4D). We also observed that GAPs were decreased in the distal colon of mice lacking goblet cells (Fig. S2D) and that LP-APC acquisition of intra-colonic fluorescent Ova was impaired in the distal colon in the absence of goblet cells and GAPs (Fig. 2f). Moreover, deletion of goblet cells impaired the induction of CD4+ T cell responses to Ova via enema in the distal colon draining $\mathrm{LN}$ in vivo (Fig. $2 \mathrm{~g}$ ). Thus, loss of goblet cells and GAPs impairs the ability of LP-APCs to acquire luminal antigen and impairs immune responses to luminal antigen in vivo despite the presence of increased intestinal leak.

Goblet cells play an important role in maintaining the intestinal barrier through mucus production and release of anti-microbial products, and accordingly deletion of goblet cells may have effects unrelated to the loss of GAPs. Therefore, to examine the role of the GAP function of goblet cells in luminal antigen delivery, we evaluated the effect of GAP inhibition on luminal antigen capture by LP-APCS and immune responses independent of deletion of goblet cells. GAPs form in response to acetylcholine (ACh) acting on the muscarinic ACh receptor 4 (mAChR4) on goblet cells, and conversely GAPs are inhibited by activation of the epidermal growth factor receptor (EGFR) in goblet cells. ${ }^{21}$ Inhibition of GAPs by luminal recombinant murine epidermal growth factor (mEGF) significantly impaired LP-APC capture of luminally administered fluorescent Ova (Fig. 3a, b), as well as the ability of LP-APCs to acquire gavaged Ova in a manner capable of inducing antigen specific CD4+ $\mathrm{T}$ cell proliferation in ex vivo cultures (Fig. 3c). Moreover, mEGF significantly impaired antigen specific CD4+ T cell responses to oral Ova in vivo in the MLN (Fig. 3d). Importantly, deletion of the EGFR in goblet cells using an inducible Math1 driven Cre recombinase, $\mathrm{EGFR}^{\mathrm{f} / \mathrm{f}}$ Math ${ }^{\mathrm{Cre} \mathrm{PR}^{*}}$ mice, reversed the effects of mEGF on GAP inhibition, and $T$ cell responses to luminal Ova in ex vivo cultures and in vivo (Fig. 3a, c, d), demonstrating that the defect in antigen capture could not be attributed to effects of EGF on LP-APCs or $\mathrm{T}$ cells. This is consistent with the effect of EGF being mediated by effecting goblet cells and GAPs. Likewise, we observed that inducible deletion of mAChR4 on goblet cells, (mAChR4 $4^{\mathrm{f} / \mathrm{f}} \mathrm{Math} 1^{\mathrm{Cr}{ }^{* P R}}$ mice) did not affect goblet cell numbers (Fig. 3e), but impaired GAP 


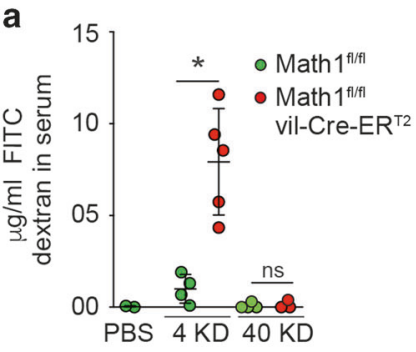

d

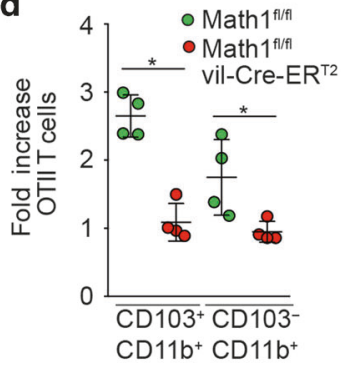

$\mathbf{f}$

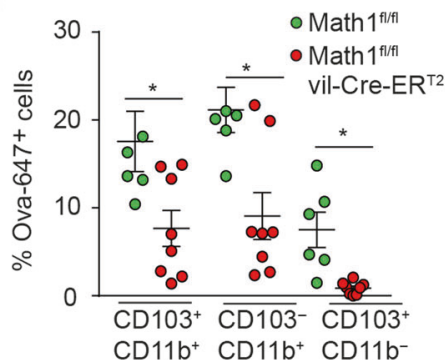

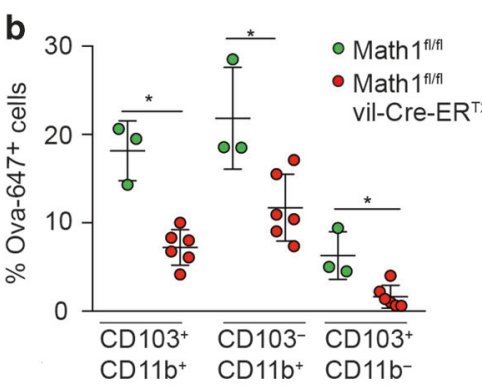

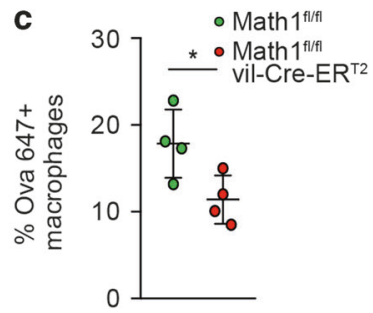

e
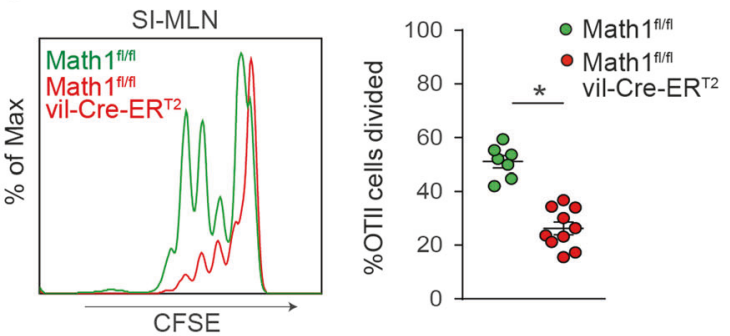

g
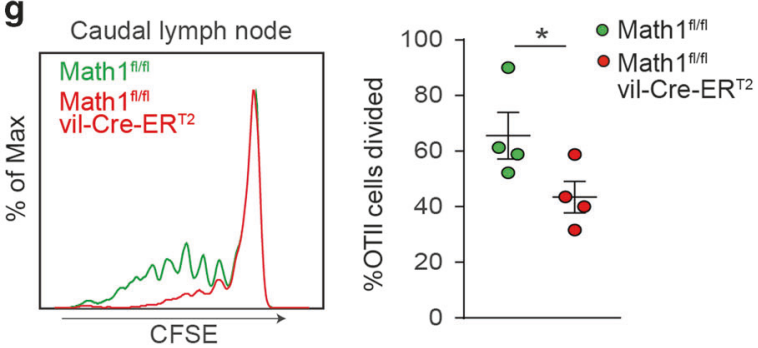

Fig. 2 Goblet cells support antigen presenting cell acquisition of luminal antigen and CD4+ T cell responses to luminal antigen in the gut draining lymph nodes. a 4kD and 40kD FITC-dextran in serum after oral gavage in Math ${ }^{\mathrm{f} / \mathrm{f}}$ vil-Cre-ER $\mathrm{VR}^{\mathrm{T2}}$ mice and Math $1^{\mathrm{f} / \mathrm{f}}$ littermate controls. b, c Luminal Ova acquisition by SI LP-APCs assessed by flow cytometry $2 \mathrm{~h}$ post oral gavage. d Antigen presentation capacity of SI LP-APCs isolated from mice Math $1^{\mathrm{f} / \mathrm{f}}$ and Math $1^{\mathrm{f} / \mathrm{f}}$ vil-Cre-ER ${ }^{\mathrm{T2}}$ mice given luminal Ova as assessed by expansion of Ova-specific OTII T cells in ex vivo cultures. e Histograms and quantification of in vivo proliferation of CFSE-labeled OTII T cells in SI draining MLN of Math ${ }^{\mathrm{f} / \mathrm{f}}$ and Math ${ }^{\mathrm{f} / \mathrm{f}}$ vil-Cre$\mathrm{ER}^{\mathrm{T2}}$ mice 2 days after oral Ova gavage. f Antigen acquisition by distal colon LP-APCs assessed by flow cytometry $2 \mathrm{~h}$ following intra-colonic administration of fluorescent Ova. g Histograms and quantification of in vivo proliferation of CFSE-labeled OTII T cells in distal colon draining caudal LN of Math $1^{\mathrm{f} / \mathrm{f}}$ and Math $1^{\mathrm{f} / \mathrm{f}}$ vil-Cre-ER ${ }^{\mathrm{T2}}$ mice 2 days after Ova enema. Data are representative of two or more replicates with $\geq 3$ mice per group, each data point represents an individual mouse. Data is presented as mean $\pm \mathrm{SEM},{ }^{*} P<0.05$, ns not significant.

formation (Fig. 3f). Unlike the deletion of goblet cells, we did not see an increase in leak when GAPs were inhibited (Fig. 3g) and accordingly we did not see a reduction in the mucus barrier when GAPs were inhibited and goblet cells remained intact (Fig. S5A and B). Inhibition of GAPs by deletion of the mAChR4 on goblet cells impaired luminal fluorescent Ova acquisition by LP-APCs (Fig. 3h. i), and impaired antigen specific CD4 $+\mathrm{T}$ cell responses to gavaged Ova in the SI draining MLN (Fig. 3j). We found that GAPs in the distal colon were inhibited by the pan-muscarinic acetylcholine receptor antagonist atropine and were induced by the $\mathrm{ACh}$ analogue carbamylcholine (Fig. S6A), but were not inhibited by deletion of mAChR4 in goblet cells (Fig. S6B), indicating that GAPs in the distal colon are induced by ACh acting on receptors other than mAChR4 and that there are yet to be identified pathways inducing GAP formation in the distal colon. While this prevented us from performing analogous studies in the distal colon to inhibit GAPs, these data support that the GAP function of goblet cells plays a role in delivering luminal antigens to LP-APCs for the induction of immune responses in the steady state.

Goblet cells and GAPs support the maintenance of Tregs and imprinting APCs in the LP

Tolerance to dietary antigens occurs in the $\mathrm{SI}$ and is mediated by CD4+ Foxp3+ pTregs that are generated in the draining LN. These
pTregs subsequently traffic to and reside in the SI LP where they are maintained by continual stimulation by LP-APCs that have acquired the cognate antigen for these pTregs from the lumen. ${ }^{1,4,11,12}$ Accordingly, these pTregs may have a limited lifespan when their cognate antigen is withdrawn. ${ }^{13}$ In contrast, a substantial proportion of the pTregs residing in the colon LP differentiate in response to microbial stimuli and are longerlived. ${ }^{13,39,40}$ A portion of these colonic pTregs can have specificity for gut bacterial antigens and their development requires GAPs in the proximal colon that are present for a defined period of time during a pre-weaning interval. ${ }^{41}$ This could suggest that luminal antigen delivery by GAP to LP-APCs might have a role in maintaining existing pTregs in the SI LP that have a more limited lifespan in the absence of continual stimulation. Indeed, we observed a decrease in the absolute number of SI LP Tregs when goblet cells/GAPs were deleted (Fig. 4a). This decrease largely affected the Helios- pTregs in the SI LP (Fig. 4b, c). We observed little change in the Helios- pTreg population in the colon LP (Fig. 4c). The relative lack of an effect of goblet cell/GAP deletion on the colonic pTreg population could reflect that adherent bacteria, which can induce immune responses by GAP independent endocytosis via enterocytes, can drive pTreg development in the colon in the steady state. ${ }^{42-44}$ Consistent with the pTregs being gut pTregs, ${ }^{39,40}$ almost all of these LP Helios- Tregs 


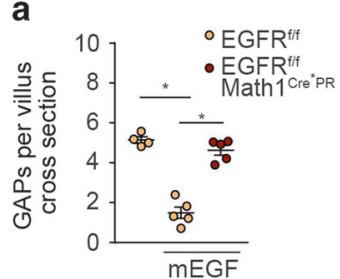

e

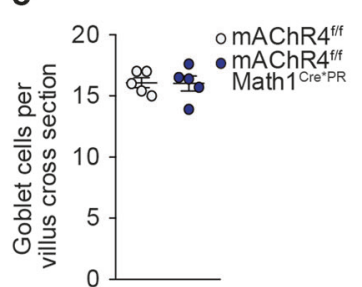

b

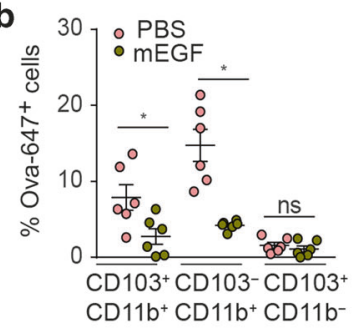

$\mathbf{f}$
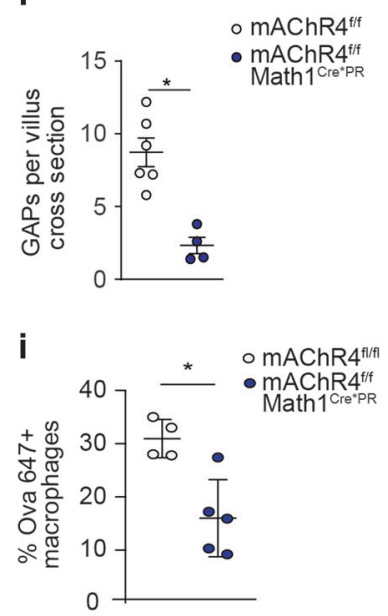
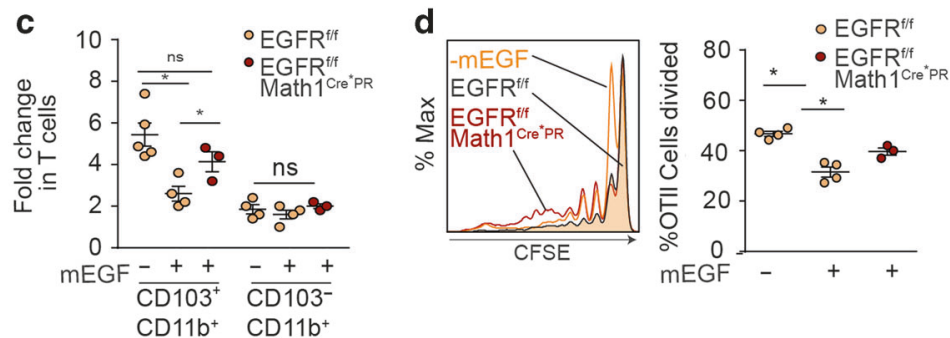

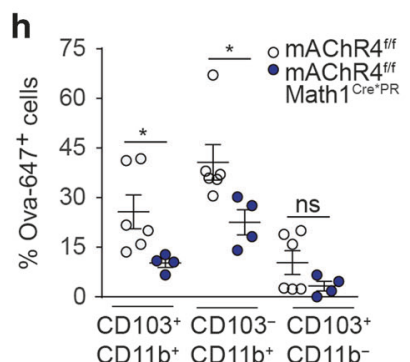

j
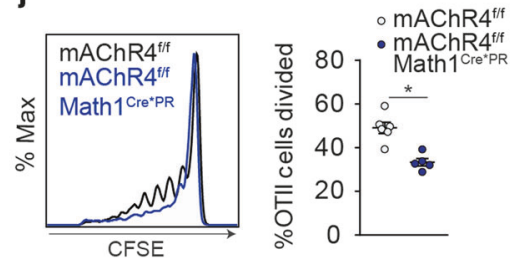

Fig. 3 The GAP function of goblet cells supports the acquisition of, and CD4 $+\mathbf{T}$ cell responses to, luminal antigen. a GAPs per villus as assessed by immunofluorescent staining, b luminal fluorescent Ova capture by LP-APCs as assessed by flow cytometry, c ability of LP-APCs to stimulate Ova-specific T cells ex vivo in response to luminal Ova, and $\mathbf{d}$ ability of Ova-specific $T$ cells to expand in vivo in response to luminal Ova in wild-type mice (panel b) and in mice lacking EGFR in goblet cells (EGFR ${ }^{\mathrm{f} / \mathrm{f}}$ Math $1^{\mathrm{Cr} \mathrm{r}^{* \mathrm{PR}}}$ mice) and littermate controls given EGF to inhibit GAPs. e Goblet cells per villus as assessed by WGA staining, $\mathbf{f}$ GAPs per villus as assessed by luminal fluorescent Ova uptake, $\mathbf{g}$ FITC-dextran $(4 \mathrm{kD})$ in serum after oral gavage, $\mathbf{h}$, $\mathbf{i}$ luminal fluorescent Ova capture by LP-APCs as assessed by flow cytometry and $\mathbf{j}$ ability of Ova-specific T cells to expand in vivo in response to luminal Ova in mice lacking mAChR4 in goblet cells (mAChR4 ${ }^{\mathrm{f} / \mathrm{f}} \mathrm{Math} 1^{\mathrm{Cre}{ }^{*} \mathrm{PR}}$ mice) and littermate controls. ${ }^{*} P<0.05$, ns not significant, data presented as the mean \pm SEM. Each data point represents an individual mouse.

expressed the transcription factor RORyt (Fig. 4b), which can be expressed by SI LP pTregs with specificity to dietary antigens. ${ }^{13}$ Further we observed that the SI LP pTreg population was reduced with GAP inhibition by $\mathrm{mEGF}$ in a goblet cell intrinsic EGFR dependent manner (Figs. 3a and 4d) and upon GAP inhibition by deletion of the mAChR4 in goblet cells (Figs. 3f and 4e), demonstrating that the GAP function of goblet cells facilitated the maintenance of SI LP pTregs.

Because the absence of GAPs impaired stimulation of Ovaspecific $T$ cells to dietary antigen in the MLN, and by extension would impair their differentiation to effector T cells or pTregs, we injected Ova intravenously to mice following adoptive transfer of OTII T cells to evaluate naive Ova-specific $T$ cell differentiation in the absence of GAPs. We observed that in the absence of goblet cells, the in vivo induction of Tregs in the MLN in response to systemic Ova was impaired (Fig. 4f). The impaired ability to induce Tregs in response to dietary Ova in mice lacking goblet cells/GAPs can in part be attributed to defects in the LP-APC population as LP-APCs isolated from mice lacking goblet cells were impaired at inducing antigen specific pTregs in ex vivo cultures (Fig. $4 \mathrm{~g}$ ).

SI LP-APCs consist of IRF8 dependent CD103+CD11b- DCs, IRF4 dependent CD103+CD11b+DCs, and CD103-CD11b+DCs and macrophages, which can express, but are not dependent upon IRF4. ${ }^{6,7}$ We observed a reduction in the CD103+CD11b+ and CD103-CD11b + populations, but not the CD103+CD11bDCs in the absence of goblet cells and GAPs (Fig. 5a). Accordingly, the absence of goblet cells and GAPs resulted in a decrease in the IRF4+ SI LP-APC population (Fig. 5b). The decrease in CD103+ CD11b+ and CD103-CD11b+ LP-APCs was dependent upon the GAP function of goblet cells as these populations were reduced in response to $\mathrm{mEGF}$ in an EGFR goblet cell dependent manner (Fig. 5c). CD103+CD11b- and CD103+CD11b+SI LP-DCs can have aldehyde dehydrogenase activity, 7,45 which facilitates the production of all-trans retinoic acid, a factor promoting the differentiation of and imprinting of pTregs with gut homing molecules. ${ }^{46-48}$ We observed that in the absence of goblet cells, SI LP-DCs had reduced aldehyde dehydrogenase (ALDH) activity (Fig. 5d) and an impaired ability to induce the expression of the gut homing molecules a4 $\beta 7$ and CCR9 on responding $T$ cells in in vitro co-cultures (Fig. 5e, f). Similar to the maintenance of preexisting LP pTregs, SI LP-DC ALDH activity was facilitated by the GAP function of goblet cells, as this was impaired by GAP inhibition by $\mathrm{mEGF}$ in a goblet cell intrinsic EGFR dependent manner (Fig. $5 \mathrm{~g}$ ) and by GAP inhibition via the deletion of mAChR4 in goblet cells (Fig. 5h).

LP macrophages have been implicated in SI LP Treg maintenance through the production of IL-10 and stimulation of pre-existing pTregs with their cognate antigen acquired from the lumen. ${ }^{11}$ We found that mice lacking goblet cells and GAPs as well as mice with goblet cells but lacking GAPs had impaired IL-10 production by $\mathrm{SI}$, but not colonic, CD11c+ MHCll + CD11b+ F4/80+ LP-APCs (Fig. 5i, j), consistent with GAPs having a role in imprinting this LP-APC subtype in the SI. Thus, goblet cells and GAPs might contribute to multiple facets of oral tolerance including antigen delivery and imprinting LP-APCs for the induction and maintenance of pTregs specific for dietary antigens.

Goblet cells and GAPs support tolerance to dietary antigen To directly evaluate the role for goblet cells and GAPs in tolerance to dietary antigens, mice lacking goblet cells and GAPs, their 

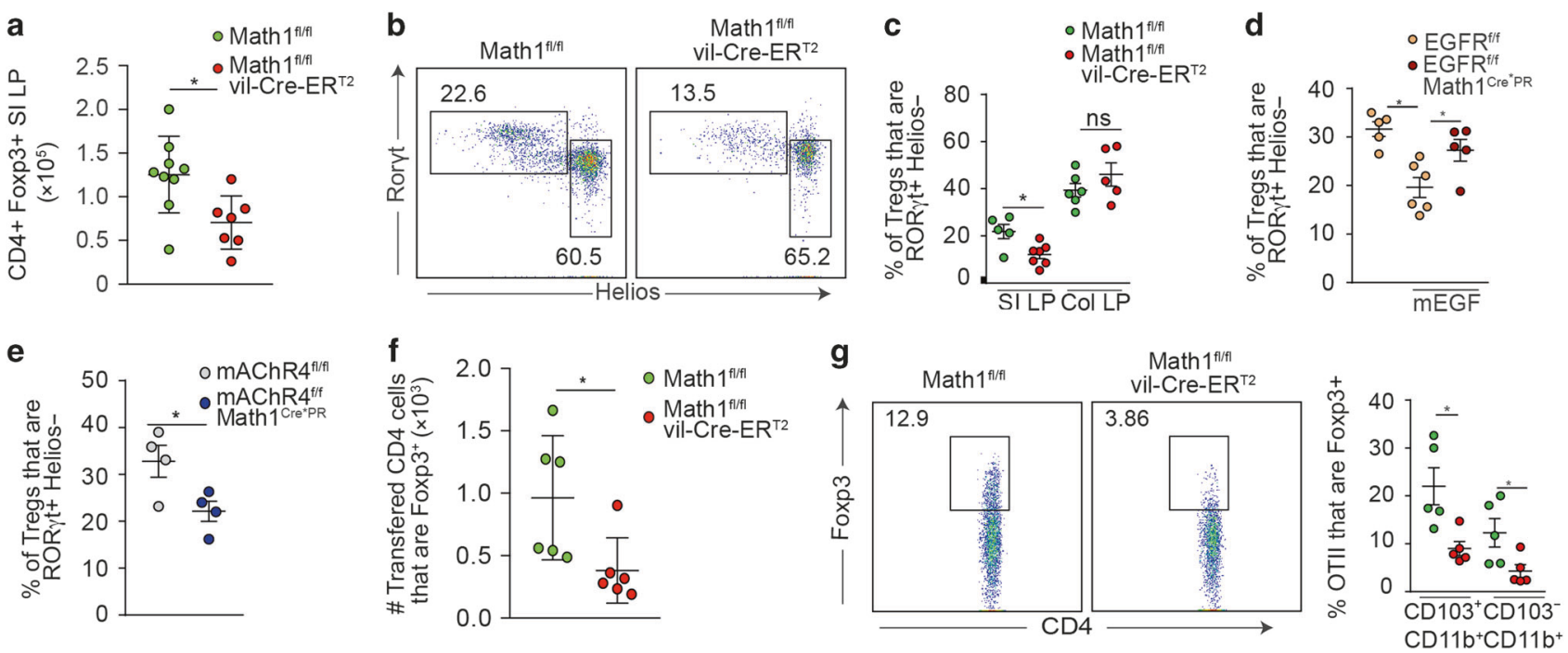

Fig. 4 GAPs support the maintenance and induction of pTregs. a Absolute numbers of SI LP Tregs, $\mathbf{b}$ flow cytometry dot plots of Helios and ROR $\gamma \mathrm{t}$ expression by CD4+ Foxp3+T cells in the SI LP, and c quantification of ROR $\gamma \mathrm{t}+$ Helios- pTregs populations in the SI and colon LP of goblet cell deficient mice (Math ${ }^{\mathrm{f} / \mathrm{f}}$ vil-Cre-ER ${ }^{\mathrm{T} 2}$ mice) and littermate controls. Quantification of SI LP ROR $\gamma \mathrm{t}+$ Helios- pTreg populations in d mice lacking EGFR in goblet cells $\left(E_{G F R}{ }^{f / f} M a t h 1{ }^{C r e}{ }^{* P R}\right.$ mice) and littermate controls treated with vehicle or mEGF, and in e mice lacking mAChR4 in goblet cells (mAChR4 $4^{\mathrm{f} / \mathrm{f}}$ Math $1^{\mathrm{Cre} \mathrm{FR}^{*}}$ mice) and littermate controls. f Quantification of Foxp3 expression by MLN OTII T cells adoptively transferred into goblet cell deficient mice and littermate controls five days following i.v. injection of Ova. g Representative flow cytometry dot plots and quantification of Foxp3 expression by OTII T cells cultured for 5 days with Ova and SI LP-APCs isolated from goblet cell deficient mice and littermate controls. ${ }^{*} P<0.05$, ns not significant. Data are presented as the mean \pm SEM. Each data point represents an individual mouse.

littermate controls, and mice in which GAPs were transiently inhibited at the time of luminal antigen administration by intraluminal mEGF or deletion of mAChR4 in goblet cells, were gavaged with Ova, immunized with Ova, challenged with Ova in the footpad, and evaluated for footpad swelling $24 \mathrm{~h}$ later. Goblet cell deficient mice and mice in which GAP formation was inhibited demonstrated significantly greater footpad swelling indicative of decreased tolerance to dietary antigen (Fig. 6a-d).

Moreover, deletion of EGFR in goblet cells at the time of $\mathrm{mEGF}$ and oral Ova administration reversed the effects mEGF on impaired tolerance, consistent with the effect of mEGF being due to GAP inhibition (Fig. 6c). Notably the Math1 Cre targets differentiated goblet cells, which turn over every 3-5 days, and therefore the inhibition of GAPs by deletion of mAChR4 and the reversal of effects of EGF by deletion of EGFR in goblet cells is largely limited to the time of luminal Ova administration, and not due to effects on goblet cells at the time of Ova immunization and challenge. While the mAChR4 independent formation of GAPs in the distal colon prevented us from directly assessing the role of the GAP function of goblet cells in tolerance to luminal antigens in the colon, we did observe that deletion of goblet cells impaired the ability to induce tolerance to Ova administered via enema (Fig. 6e). Loss of goblet cells might induce inflammatory responses due to the deficient mucus barrier, which could affect the capture of luminal substances by resident LP-APCs and the induction of tolerance independent of the loss of GAPs. Indeed, we observed that deletion of goblet cells resulted in an increase in monocytes and neutrophils in the SI lamina propria (Fig. S7A). However, we did not see an increase in monocytes in the lamina propria when GAPs were inhibited and goblet cells remained intact (Fig. S7B) suggesting that inflammatory responses alone do not account for the loss of tolerance when GAPs are inhibited. Mice with goblet cell and GAP manipulation had increased serum levels of interferon- $\gamma$ (IFN $\gamma$ ) following immunization (Fig. $6 f-i)$, correlating with their loss of tolerance to dietary Ova. The impaired tolerance in the absence of goblet cells or GAPs was not as severe as that seen in the absence of luminal Ova exposure (Fig. 6a-e), suggesting the potential for other or compensatory routes of luminal antigen delivery in the absence of goblet cells and GAPs. However, in total these observations indicate that GAPs support the induction of tolerance to luminal antigens on multiple levels.

\section{DISCUSSION}

The gut lumen contains trillions of microbes and abundant microbial products. Inducing and maintaining tolerance to innocuous substances originating from this potentially inhospitable environment is fundamental to maintaining homeostasis and health. Indeed, tolerance is so effectively induced to antigens originating from the gut lumen that oral tolerance regimens are being leveraged to treat extra-intestinal diseases. ${ }^{49-51}$ Accordingly, how tolerance is induced and maintained at this mucosal surface has been a topic of many studies.

The gut microenvironment has unique properties supporting tolerance. Tolerance to non-self antigens is largely mediated by the conversion of naive T cells into Foxp3 expressing pTregs, ${ }^{52,53}$ which is facilitated by a local environment containing all-trans retinoic acid (ATRA) and TGF $3{ }^{46,47,54,55}$ Within the gut CD103+ DCs and MLN stromal cells expressing retinaldehyde dehydrogenase, the enzyme necessary to convert retinal to the biologically active ATRA, are sources of ATRA supporting pTreg induction and imprinting gut homing molecules on lymphocytes. ${ }^{46,56-60}$ DC imprinting with retinaldehyde dehydrogenase activity is induced by luminal retinoids and by DC association with the intestinal epithelium. ${ }^{45,61,62}$ Moreover, the goblet cell protein, mucin 2, promotes tolerogenic properties in DCs inducing pTregs including the production of TGF $\beta$ and the expression of retinaldehyde dehydrogenase, $^{63}$ and select members of the gut microbiota promote pTregs through bacterial products or metabolites. ${ }^{42,64-70}$ Thus, these unique properties contribute to the tolerogenic tone of the gut environment, yet how luminal antigens are acquired by the immune system for the induction of tolerance and whether this process contributes to tolerance beyond antigen capture have been unexplored.

How luminal antigens are encountered by the immune system may affect the phenotype of the subsequent immune response. ${ }^{71-73}$ 
a

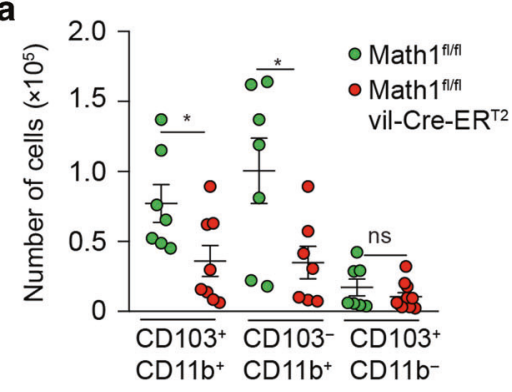

b

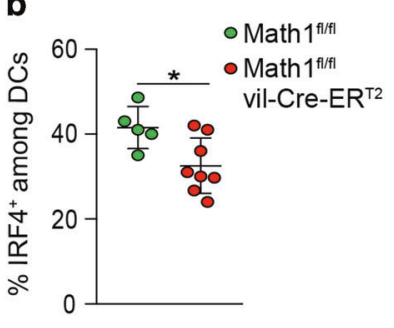

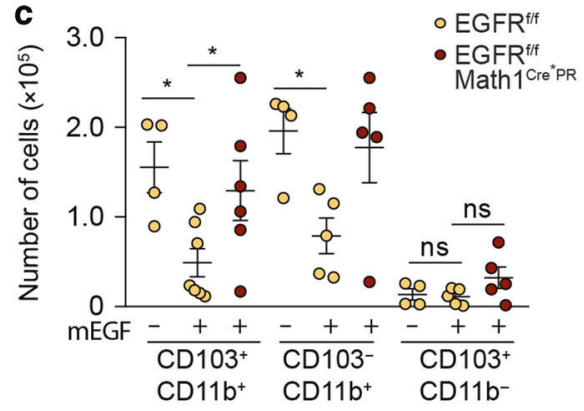

d

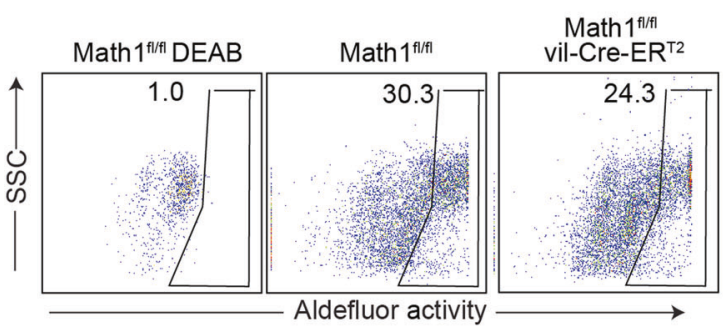

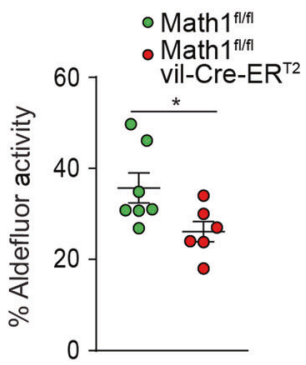

e

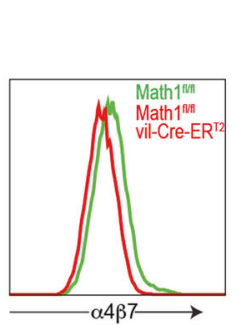

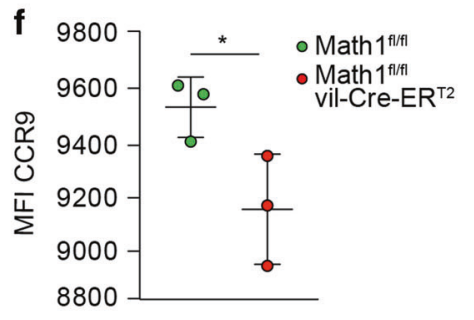

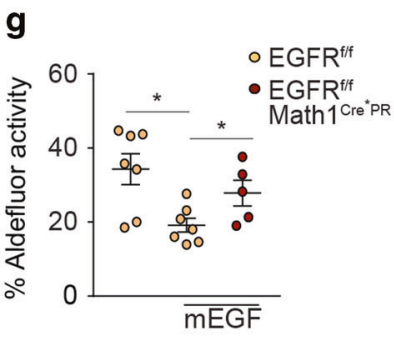

h

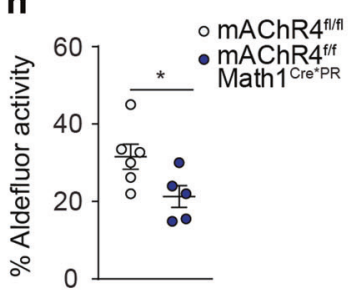

i

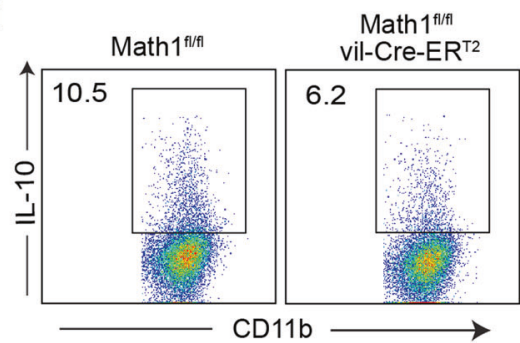

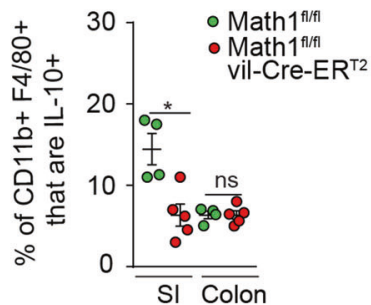

j

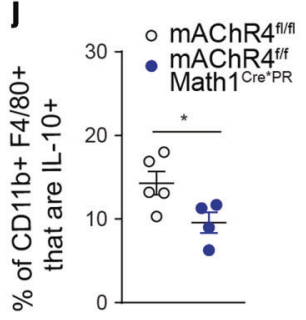

Fig. 5 GAPs support the imprinting of LP-APCs. Quantification of a SI LP-APC subsets and $\mathbf{b}$ IRF4+ SI LP-DCs in goblet cell deficient mice (Math $1^{\mathrm{f} / \mathrm{f}}$ vil-Cre-ER ${ }^{\mathrm{T2}}$ mice) and littermate controls. c Quantification of SI LP-APC subsets in mice lacking EGFR in goblet cells (EGFR ${ }^{\mathrm{f} / \mathrm{f}}$ Math $1^{\mathrm{Cre}^{*} P R}$ mice) and littermate controls treated with mEGF. d Flow cytometry dot plots and quantification of aldehyde dehydrogenase (ALDH) activity in $\mathrm{LP} C D 11 \mathrm{c}+\mathrm{MHCII}+\mathrm{SI}$ APCs from goblet cell deficient mice (Math $1^{\mathrm{f} / \mathrm{f}}$ vil-Cre-ER ${ }^{\mathrm{T2}}$ mice) and littermate controls. Expression of gut homing molecules e $\alpha 4 \beta 7$ and $\mathbf{f}$ CCR9 on OTII T cells following three days of in vitro culture with Ova and CD103+ DCs isolated from goblet cell deficient mice or littermate controls. Quantification of SI LP-APC with ALDH activity in $\mathbf{g}$ mice lacking EGFR in goblet cells (EGFR ${ }^{\mathrm{f} / \mathrm{f}} \mathrm{Math}_{\mathrm{Cr}} \mathrm{Cre}^{*} \mathrm{PR}$ mice) and littermate controls treated with $\mathrm{mEGF}$ and in $\mathbf{h}$ SI LP-APCs from mice lacking mAChR4 in goblet cells (mAChR4 ${ }^{\mathrm{f} / \mathrm{f}} \mathrm{Math} 1^{\mathrm{Cre} \mathrm{PRR}^{*}} \mathrm{mice}^{\mathrm{m}}$ and littermate controls. i Flow cytometry plots of SI LP macrophages and quantification of IL-10 expression by LP CD45+ CD11C+ MHCII+ F480+ cells from mice lacking goblet cells and their littermate controls. $j$ Quantification of IL-10 expression by SI LP macrophages from mice lacking mAChR4 in goblet cells and their littermate controls. ${ }^{*} P<0.05$, ns not significant. Data is presented as the mean \pm SEM. Each data point represents and individual mouse, with the exception of $\mathbf{e}$, $\mathbf{f}$, where LP-APCs were pooled from three goblet cell deficient mice or three littermate controls.

A landmark discovery identified that LP-APCs had the ability to extend dendrites between epithelial cells to capture luminal bacteria without compromising the epithelial barrier, ${ }^{16,74}$ suggesting that this process might allow minimally disruptive direct capture of luminal substances. However, LP-APC extension of TEDs is absent in some mouse strains, ${ }^{24}$ suggesting that unlike oral tolerance, LP- APC TED extension is not a universal phenomenon and other pathways of luminal antigen capture inducing oral tolerance exist. In addition, while the extension of TEDs is impaired in the absence of $\mathrm{CX} 3 \mathrm{CR} 1,{ }^{15} \mathrm{CD} 4+\mathrm{T}$ cell responses to luminal antigens are not, ${ }^{4,15}$ suggesting that the defect in oral tolerance in $\mathrm{CX}_{3} \mathrm{CR} 1$-deficient mice was unrelated to luminal antigen capture. We observed that the extension of TEDs by LP-APCs is very rare in the steady state but became more common after the removal of the luminal contents and mucus layer, occurring in a frequency similar to prior reports. ${ }^{15,25}$ Why removal of the luminal contents and mucus layer induces TED extension is unclear, but could be related to the release of lactate and pyruvate by stressed epithelial 

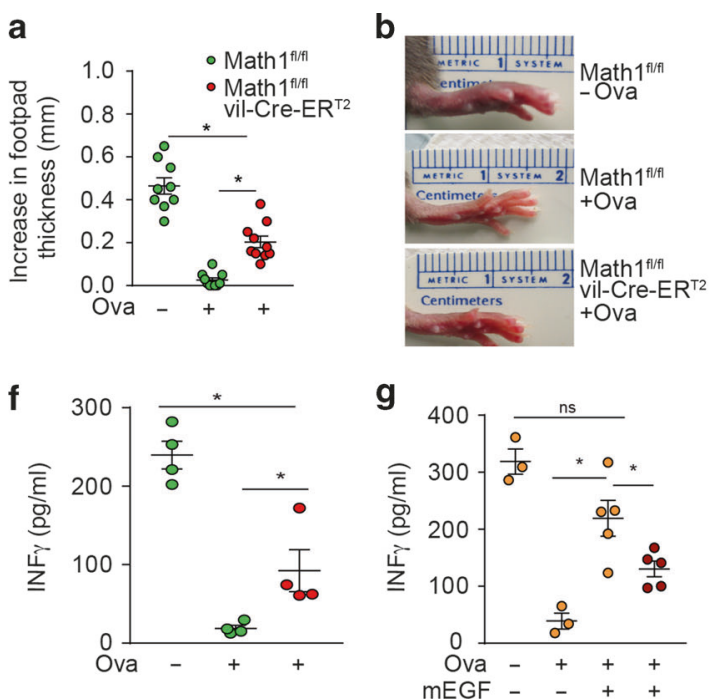
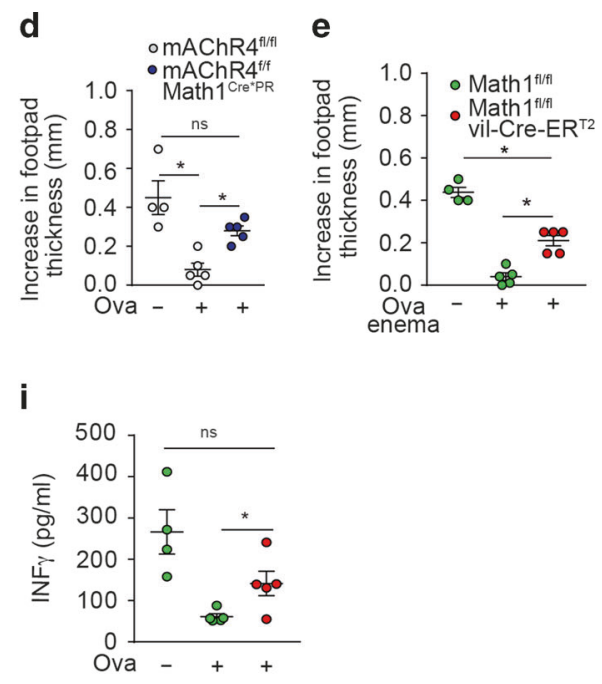

Fig. 6 GAPs support tolerance to dietary antigen in the SI and tolerance to luminal antigens in the distal colon. a Quantification and b images of footpad swelling following the induction of oral tolerance by dietary Ova, Ova immunization, and Ova footpad challenge in mice lacking goblet cells (Math $1^{\mathrm{ft} / \mathrm{f}}$ vil-Cre-ER ${ }^{\mathrm{T2}}$ mice) and their littermate controls. Quantification of footpad swelling following dietary Ova, Ova immunization, and Ova footpad challenge in c mice lacking EGFR in goblet cells (EGFR ${ }^{\mathrm{f} / \mathrm{f}} \mathrm{Math} 1^{\mathrm{Cre}{ }^{*} \mathrm{PR}}$ mice) and their littermate controls treated with mEGF and in d mice lacking mAChR4 in goblet cells (mAChR4 ${ }^{T / f}$ Math $1^{\text {Cre }}{ }^{* P R}$ mice) and littermate controls. e Quantification of footpad swelling following the induction of tolerance by Ova enema, Ova immunization, and Ova footpad challenge in mice lacking goblet cells

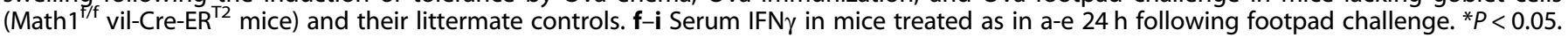
Data are presented as the mean \pm SEM. Each data point represents an individual mouse.

cells as these metabolites were recently identified to induce TED extension in $\mathrm{CX}_{3} \mathrm{CR} 1+\mathrm{LP}_{-} \mathrm{APCs}{ }^{75}$ and we have observed that TED extension occurs when mice expire while imaging under anesthesia in the absence of removal of the luminal contents and mucus layer (unpublished observation). We did not observe LP-APC TED extension in the duodenum, the site where gavaged antigen is acquired by $\mathrm{CX}_{3} \mathrm{CR} 1+\mathrm{LP}-\mathrm{APCs},{ }^{26}$ or in the distal colon, the site where tolerance to luminal antigen is induced in the colon. ${ }^{14}$ While it is impossible to exclude a contribution of LP-APC TED extension, combined with the above observations, these findings indicate that LP-APC TED extension is less likely to be a major route of steady state soluble luminal antigen capture for the induction of oral tolerance.

Early observations suggested that $M$ cells were restricted to the epithelium overlying the Peyer's patches; however, subsequent studies identified $M$ cells overlying the non-follicle bearing villous epithelium. ${ }^{18}$ Villous $M$ cells are rare in the steady state but can be induced by systemic treatment with TNF superfamily member receptor activator of NF-K $\beta$ ligand (RANKL), whose expression is normally restricted to subepithelial stromal cells restricted to the Peyer's patches. ${ }^{76}$ These villous $M$ cells can be closely associated with mononuclear cells and have the capacity to transcytose bacteria to induce immune responses to luminal bacteria. ${ }^{18,77} \mathrm{We}$ also found villous $M$ cells to be very rare in the steady state, suggesting they are not a major pathway for luminal antigens to traverse the epithelium to support oral tolerance. Similarly, barrier leak, as evidenced by the presence of luminally administered $4 \mathrm{kD}$ dextran in the serum did not correlate with LP-APCs acquisition of luminal antigen. Why barrier leak is less effective at loading LPAPCs with antigen in a manner capable of inducing $T$ cell responses is unclear but could be related to the size of substances delivered via paracellular leak relative to the size of proteins/ polypeptides required to induce antigen specific $T$ cell responses as we did not see an increase in $40 \mathrm{kD}$ dextran in the serum following gavage in goblet cell deficient mice.

In contrast, the presence of intestinal epithelial cells filling with luminal antigen was common in the steady state. Consistent with a recent report, ${ }^{27}$ we observed enteroendocrine cells containing luminal antigen; however, they were rare and limited to the duodenum in the steady state. We more commonly observed Paneth cells containing luminal antigen, but these were still relatively rare occurring on average in one Paneth cell in every two crypt cross sections. Moreover, Paneth cells are less likely to be a major contributor to steady state luminal antigen delivery supporting tolerance as they are absent from the colon, and due to their longer lifespan, persist for weeks following deletion of Math1 in epithelial cells, which we observed results in significantly impaired luminal antigen delivery to LP-APCs. In contrast, goblet cells filling with luminal antigen were commonly observed in the regions of the gut where luminal antigens are acquired to induce tolerance, suggesting that goblet cells and GAPs may be pathways delivering dietary antigens to LP-APCs to support oral tolerance. Of note GAPs are present in strains of mice in which the extension of TEDs by LP-APCs are absent. ${ }^{20,24}$ Indeed, we observed that in the absence of goblet cells and GAPs luminal antigen capture by CD103+CD11b+ , CD103-CD11b+, and CD103+CD11b- LPAPCs was impaired. Our initial observation of GAP mediated antigen delivery to LP-APCs reported that GAPs delivered antigen to CD103+ LP-DCs. ${ }^{20}$ These studies focused on the functional outcome of inducing antigen specific $T$ cell responses to luminal antigen, which was largely limited to the CD103 + LP-DC population and was impaired in the absence of goblet cells. ${ }^{20}$ However, other APC populations could acquire luminal antigen and stimulate antigen specific $T$ cell responses when GAPs were induced above baseline levels, ${ }^{20}$ suggesting that GAPs deliver antigen to these APC populations as well. We have observed CD103- LP-APC populations interacting with GAPs in the SI and colon. ${ }^{78,79}$ The preferential ability of CD103+ LP-APCs over CD103 - LP-APCs to induce T cell responses to luminal antigen may be related to their enhanced antigen presentation and stimulation capacities ${ }^{4}$ or may be due to passage of antigen from CD103- LP-APCs to CD103+ LP-DCs. ${ }^{26}$ Irrespective of the pathway by which CD103+ LP-DCs acquire luminal antigen, either by direct capture from goblet cells, or from transfer from CD103- LP-APCs, our observations indicate that this process is supported by the GAP function of goblet cells. While luminal antigen capture by 
LP-APCs was nearly undetectable in the absence of GAPs, proliferation of dietary antigen specific T cells in the MLN and oral tolerance, as measured by DTH responses, were less dramatically impaired. This could be consistent with contributions of dietary antigen capture at other sites, such as the Peyer's patches, to $T$ cell responses in the MLN and tolerance to dietary antigens.

The findings presented here indicate that GAPs function beyond simple antigen delivery to promote oral tolerance. LP-DCs with ALDH activity produce all-trans retinoic acid, ${ }^{45}$ which promotes the induction of pTregs. ${ }^{4,46,47,63}$ Further, the production of IL-10 by resident $L P$ macrophages supports the expansion and maintenance of pre-existing LP pTregs specific for dietary antigens. ${ }^{11}$ We found that in the absence of goblet cells/GAPs imprinting SI LP-APCs with ALDH activity and the production of IL-10 by SI macrophages were impaired. When CD103+ LP-DCs acquire luminal antigens from GAPs, they also acquire goblet cell proteins. ${ }^{20}$ Combined with observations that the goblet cell protein mucin 2 imprints DCs with ALDH activity, ${ }^{63}$ this suggests that CD103+ LP-DC imprinting by GAPs may occur during antigen acquisition and that GAPs may deliver tolerogenic signals in concert with luminal substances to support antigen specific tolerance induction. The mechanism of tolerance induction to luminal substance encountered in the distal colon differs from that of the $\mathrm{SI}$ and can utilize other APC populations. ${ }^{8,14}$ While we did not observe LP-APCs defects in the colon in the absence of goblet cells/GAPs, the relevant properties of the APCs inducing tolerance in the distal colon are not known, and accordingly whether GAPs in the distal colon play an analogous role influencing this APC phenotype remains to be investigated. Beyond this we noted that GAPs supported the maintenance of pre-existing pTregs in the SI LP. These pTregs regressed within days of GAP inhibition, a time course that is much faster than regression of SI LP pTregs when deprived of cognate antigen. ${ }^{13}$ This suggests that GAPs may play additional yet to be identified roles beyond antigen delivery in shaping the immune landscape of the gut. Related to this, enteric viral infection abrogates oral tolerance and promotes Th1 immune responses to dietary antigen, ${ }^{80}$ and enteric bacterial infection inhibits GAPs and shifts immune responses to dietary antigen away from tolerance toward Th17 responses. ${ }^{81}$ In the context of the findings presented here, this might suggest that GAP inhibition during enteric infection is a physiologic response facilitating inflammatory responses for pathogen clearance.

Immune tolerance to innocuous substances encountered in the gut lumen is a recognized phenomenon that is essential for gut health. How this process occurs is a fundamental question. Here we identify a role for goblet cells and GAPs as routes for luminal antigen encounter by the immune system for the induction of tolerance to dietary antigens in the steady state. Moreover, we observed that GAPs imprint LP-APCs with properties necessary for the induction and maintenance of pTregs. Combined with studies demonstrating that GAP formation is closely regulated to prevent inappropriate inflammatory responses to luminal substances encountered in hostile settings, ${ }^{21,78,81}$ and that GAPs promote the induction of antigen specific tolerance to commensal bacteria during a defined pre-weaning interval, ${ }^{41}$ the observations presented here suggest that goblet cell and GAP dysfunction may contribute to the pathogenesis of intestinal inflammatory diseases. Moreover, these observations suggest that restoring goblet cell and GAP function may be one component of approaches to restore gut immune homeostasis.

\section{METHODS}

See online supplementary information for complete methods.

Mice

All mice were 10 or more generations on the $\mathrm{C} 57 \mathrm{BL} / 6$ background, with the exception of the mAChR4 $4^{\mathrm{fl} / \mathrm{fl}}$ mice, which were 6-7 generations on the C57BL/6 background at the time of these studies. C57BL/6 mice, congenic CD45.1 B6SJL mice, OTII T cell receptor transgenic mice, ${ }^{82} \mathrm{CD} 11 \mathrm{c}^{\mathrm{YFP}}$ transgenic mice, ${ }^{83}$ $\mathrm{CX}_{3} \mathrm{CR} 1^{\mathrm{GFP}}$ mice, $^{84} \mathrm{Math}^{\mathrm{fl} / \mathrm{fl}}$ mice, $^{33}$ FoxP3 ${ }^{\mathrm{GFP}}$ mice, $^{85}$ were purchased from The Jackson Laboratory (Bar Harbor, ME) or The National Cancer Institute (Frederick, MD). Transgenic mice in which a tamoxifen-dependent Cre recombinase is expressed under the control of the villin promoter (vil-Cre-ERT2) mice ${ }^{86}$ were a gift from Sylvie Robine (Institut Curie, Paris, France). Math $1^{\mathrm{fl} / \mathrm{fl}}$ mice were bred to vil-Cre-ER ${ }^{\mathrm{T2}}$ mice to generate mice with inducible depletion of goblet cells following deletion of Math1 in villin expressing cells. Math $1^{\mathrm{f} / \mathrm{fl}}$ vil-Cre-ER ${ }^{\mathrm{T} 2}$ mice and the injection protocol to induce goblet cell deletion have been previously described. ${ }^{21}$ EGFR $^{\text {fl/f }}$ mice ${ }^{87}$ were a gift from Dr. David Threadgill, University of North Carolina. mAChR4 ${ }^{\mathrm{f} / \mathrm{fl}}$ mice $^{88}$ were a kind gift from Jurgen Wess (National Institute Health, Bethesda, MD). $\mathrm{EGFR}^{\mathrm{fl} / \mathrm{fl}}$ mice and $\mathrm{mAChR} 4^{\mathrm{f} / \mathrm{fl}}$ mice were bred to Math1 ${ }^{\mathrm{Cre}{ }^{* P R}}$ mice ${ }^{35}$ to generate mice with an inducible deletion of EGFR or mAChR4 in goblet cells. Mice were housed in a specific-pathogenfree facility and fed routine chow diet. Mice of both sexes were used in this study. Animal procedures and protocols were performed in accordance with the IACUC at Washington University School of Medicine.

Intravital two-photon (2 P) microscopy

In vivo two-photon imaging was performed as previously described. ${ }^{20}$

Evaluation of luminal antigen uptake by epithelial cells Tetramethylrhodamine-labeled $10 \mathrm{kD}$ dextran or Texas Red labeled ovalbumin was administered in the SI, proximal and distal colon of anesthetized mice. After $1 \mathrm{~h}$, mice were sacrificed, and tissues thoroughly washed with cold PBS before fixing in $10 \%$ formalin buffered solution. Tissues were embedded in optimal cutting temperature compound (Fisher Scientific, Pittsburgh, PA) and $6 \mu \mathrm{m}$ sections prepared. For studies in Fig. 1, sections were stained with wheat germ agglutinin (WGA), Ulex europaeus agglutinin I (UEA I), anti-lysozyme antibodies, or anti-chromogranin A antibodies to identify goblet cells in the SI, goblet cells in the colon, Paneth cells, and enteroendocrine cells respectively. Sections were then stained with 4',6-diamidino-2-phenylindole (DAPI, Sigma-Aldrich, St Louis, MO) and imaged using an Axioskop 2 microscope with a Plan-Neofluar $\times 20 / 0.5$ objective (Carl Zeiss Microscopy, Thornwood, NY).

Analysis of luminal fluorescent antigen uptake by LP-APCs Mice were anesthetized and $200 \mu \mathrm{g}$ of Alexa Fluor 647 labeled ovalbumin (Ova-A647), dissolved in phosphate buffered saline (PBS), or PBS alone (controls), was injected into the SI lumen, or given via enema using a $16 \mathrm{G}$ plastic cannula inserted $3 \mathrm{~cm}$ transanal into the colon. In some experiments, anesthetized mice were treated intraluminally with $10 \mu \mathrm{g}$ murine EGF (Shenandoah Biotechnology, Warwick, PA) dissolved in PBS, or PBS alone 20 min prior to Ova-A647 administration. Two hours later cellular populations were isolated from the non-Peyer's patch bearing SI or distal colon as described previously. ${ }^{45}$ The distal colon segment represents the last two $\mathrm{cm}$ of the colon. Isolated LP cells were stained for APC markers and evaluated for Ova-A647 positive staining by flow cytometry.

Analysis of luminal antigen delivery to LP-APCs and induction of T cell proliferation in vitro

Mice were anesthetized and $2 \mathrm{mg}$ of ovalbumin (Ova) dissolved in phosphate buffered saline (PBS), or PBS alone (controls), was injected intraluminally into the SI. For delivery of Ova by enema or a $16 \mathrm{~g}$ plastic cannula was inserted $3 \mathrm{~cm}$ transanal into the colon. In some experiments, anesthetized mice were intraluminally treated with $10 \mu \mathrm{g}$ murine EGF (Shenandoah Biotechnology, 
Warwick, PA) 20 min prior to Ova administration. Two hours later cellular populations were isolated from the non-Peyer's patch bearing SI LP. APC populations and Ova-specific CD4+ OTII T cells were isolated with flow-cytometric cell sorting and cultured at a ratio of 1:10 APCs $\left(1 \times 10^{4}\right)$ to T cells $\left(1 \times 10^{5}\right)$. As a positive control, $10 \mu \mathrm{g}$ Ova was added to cultures of APC populations isolated from mice receiving luminal PBS. After 3 days, cultures were evaluated for the number of $T$ cells by flow cytometry and cell counting.

Adoptive $\mathrm{T}$ cell transfer and analysis of in vivo antigen specific $\mathrm{T}$ cell responses to luminal Ova

To evaluate the role of goblet cells and GAPs on delivery of luminal antigen and antigen specific $T$ cell proliferation in the draining lymph nodes, single-cell suspensions of Ova-specific $T$ cells were prepared from spleens and MLNs of CD45.1 $1^{+}$OTII T cell receptor transgenic mice, and CD4 T cell enrichment was performed using magnetic beads (Stemcell Technology, Vancouver, BC). Enriched $\mathrm{CD}^{+}{ }^{\mathrm{T}}$ cells were labeled with $2 \mu \mathrm{M}$ CFSE (Invitrogen, Carlsbad, CA) and $2 \times 10^{6}$ CFSE-labeled cells were i.v. transferred into sex matched recipient mice. Twenty-four hours after transfer, mice were orally gavaged with $15 \mathrm{mg}$ Ova (Sigma-Aldrich, St. Louis, MO) or in some experiments mice were administered $25 \mathrm{mg}$ of ovalbumin via enema using a $16 \mathrm{G}$ plastic cannula as above. $\mathrm{EGFR}^{\mathrm{fl} / \mathrm{fl}}$ or $\mathrm{EGFR}^{\mathrm{fl} /}$ ${ }^{f l}$ Math ${ }^{C{ }^{C{ }^{*} P R}}$ mice, were administered with $10 \mu \mathrm{g}$ of murine EGF 20 min prior to receiving $15 \mathrm{mg}$ ovalbumin in saline orally. Two days later SI draining MLNs or distal colon draining caudal and iliac LNs were removed and single-cell suspensions were prepared and analyzed by flow cytometry for CD45.1, CD3, V 35 , Va2, and CSFE. To evaluate the effect of systemic antigen administration on transferred T cells, $24 \mathrm{~h}$ post adoptive transfer $200 \mu \mathrm{g}$ of Ova was administered i.v. and transferred $\mathrm{T}$ cells evaluated on the same schedule as described above.

pTreg generation in vivo and in vitro

To evaluate de novo induction of pTreg cells, single-cell suspensions from spleen and MLNs from Ova-specific CD45.1 $1^{+}$ Foxp3 ${ }^{\text {GFP }}$ OTII T cell receptor transgenic mice were flow cytometrically sorted for $\mathrm{GFP}^{-}, \mathrm{V} \beta 5^{+}, \mathrm{Va}^{+}, \mathrm{CD} 45.1^{+}, \mathrm{CD} 62^{\text {hi }}$ cells. $5 \times$ $10^{5}$ cells were i.v. administered into recipient Math $1^{\mathrm{f} / \mathrm{f}}$ vil-Cre-ER ${ }^{\mathrm{T}}$ or Math $1^{\mathrm{f} / \mathrm{fl}}$ mice 7 days after start of tamoxifen treatment. Recipient mice were gavaged with $15 \mathrm{mg} \mathrm{Ova,} \mathrm{and} \mathrm{SI} \mathrm{draining}$ MLNs were evaluated five days later for Foxp $3^{\text {GFP+ }}$ cells among the transferred cells. To evaluate the de novo generation of pTregs in vitro naive Foxp3 ${ }^{\text {GFP- }}$ CD45.1 OTII T cells were isolated as above and cultured with flow cytometrically sorted LP-APCs at a ratio of 10:1 with $40 \mu \mathrm{g}$ of exogenous Ova. Five days later cultures were harvested and evaluated for Foxp $3^{\mathrm{GFP}+}$ expression by T cells.

ALDH activity

To evaluate the expression of ALDH in DCs, intestinal LP cells were stained using ALDEFLUOR (StemCell Technologies, Vancouver, BC, Canada) per the manufacturer's recommendations as previously described. ${ }^{45}$

Analysis of CCR9 and $a 4 \beta 7$ induction by $T$ cell in vitro Cellular populations were isolated from the non-Peyer's patch bearing SI LP of mice lacking goblet cells and littermate controls. $\mathrm{CD} 11 \mathrm{c}+\mathrm{MHCll}+\mathrm{CD} 103+\mathrm{CD} 11 \mathrm{~b}+$ populations and Ova-specific CD4+ OTII T cells were isolated with flow-cytometric cell sorting. Cell were cultured at a ratio of 1:10 APCs $\left(1 \times 10^{4}\right)$ to T cells $(1 \times$ $10^{5}$ ) and $2 \mu \mathrm{g}$ Ova was added each well. After 3 days, cultures were evaluated for the expression of CCR9 and $a 4 \beta 7$ on T cells by flow cytometry.

Measurement of mucus thickness

To determine the thickness of mucus layer, SI tissue containing luminal matter were fixed in Carnoy's fixative overnight. Subsequently, tissues were passed reducing concentration of methanol, before being embedded in OCT. Tissue sections were cut to a thickness of $6 \mu \mathrm{m}$ and slides were dried to room temperature before staining with Alcian Blue for mucus.

Oral tolerance and delayed type hypersensitivity responses Mice were given Ova $20 \mathrm{~g} / \mathrm{L}$ in drinking water, or drinking water alone for 2 weeks, or alternatively were gavaged with $20 \mathrm{mg}$ Ova daily for 2 weeks concurrent with gavage of $10 \mu \mathrm{g}$ murine EGF or given $25 \mathrm{mg}$ Ova via enema. Two weeks and four weeks following dietary Ova exposure mice were immunized subcutaneously with $100 \mu \mathrm{g}$ Ova in incomplete Freund's Adjuvant (Sigma-Aldrich). Two weeks after the last immunization mice were challenged with 20 $\mu \mathrm{g}$ Ova in the footpad and the change in footpad thickness evaluated using measurements taken with micrometer calipers before and $24 \mathrm{~h}$ after challenge. Blood was collected $24 \mathrm{~h}$ after footpad challenge and serum levels of IFN $\gamma$ were measured using Mouse IFNY ELISA kit (eBioscience, San Diego, CA), according to manufacturer's protocol.

Statistical analysis

Data analysis using a two sided student's $t$-test for studies involving two groups or one way ANOVA with a Dunnett's or Tukey's post-test with correction for multiple comparisons for studies involving three or more groups was performed using GraphPad Prism (GraphPad Software Inc., San Diego, CA). A cut-off of $p<0.05$ was used for significance.

\section{ACKNOWLEDGEMENTS}

Supported by grants: DK097317, Al131342, Al112626, DK109006, Al136515, Al 140755, and Crohn's and Colitis Foundation Research Fellowship Award 348359 and Swedish Research Council International Postdoc Award 2014-00366. The authors wish to thank Mark J Miller for advice and assistance with in vivo two-photon imaging. The Washington University Digestive Diseases Research Center Core, supported by $\mathrm{NIH}$ grant P30 DK052574 assisted with imaging. Two photon in vivo imaging was performed at the Washington University School of Medicine In Vivo Imaging Core. The High Speed Cell Sorter Core at the Alvin J. Siteman Cancer Center at Washington University School of Medicine and Barnes-Jewish Hospital in St. Louis, MO. provided flow-cytometric cell sorting services. The Siteman Cancer Center is supported in part by NCl Cancer Center Support Grant P30 CA91842.

\section{AUTHOR CONTRIBUTIONS}

D.H.K., J.K.G., K.A.K., K.G.M., A.N.F., S.S.B., and J.E.D. performed the experiments. D.H.K., J.K.G., K.G.M., S.P.H., C.S.H., and R.D.N. designed the study. D.H.K., J.K.G., S.P.H., C.S.H., and R.D.N. wrote the manuscript. All authors have reviewed and agree with the manuscript content.

\section{ADDITIONAL INFORMATION}

The online version of this article (https://doi.org/10.1038/s41385-019-0240-7) contains supplementary material, which is available to authorized users.

Competing interests: R.D.N., K.A.K., and K.G.M. are inventors on U.S. Nonprovisional Application Serial No. 15/880,658 Compositions And Methods For Modulation Of Dietary And Microbial Exposure.

Publisher's note Springer Nature remains neutral with regard to jurisdictional claims in published maps and institutional affiliations.

\section{REFERENCES}

1. Pabst, O. \& Mowat, A. M. Oral tolerance to food protein. Mucosal Immunol. 5, 232-239 (2012).

2. Bogunovic, M. et al. Origin of the lamina propria dendritic cell network. Immunity 31, 513-525 (2009).

3. Varol, C. et al. Intestinal lamina propria dendritic cell subsets have different origin and functions. Immunity 31, 502-512 (2009).

4. Schulz, O. et al. Intestinal CD103+, but not CX3CR1+, antigen sampling cells migrate in lymph and serve classical dendritic cell functions. J. Exp. Med. 206, 3101-3114 (2009). 
5. Persson, E. K. et al. IRF4 transcription-factor-dependent CD103(+)CD11b(+) dendritic cells drive mucosal $\mathrm{T}$ helper 17 cell differentiation. Immunity $\mathbf{3 8}$ 958-969 (2013)

6. Schlitzer, A. et al. IRF4 transcription factor-dependent CD11b+ dendritic cells in human and mouse control mucosal IL-17 cytokine responses. Immunity $\mathbf{3 8}$, 970-983 (2013)

7. Luda, K. M. et al. IRF8 transcription-factor-dependent classical dendritic cells are essential for intestinal T cell homeostasis. Immunity 44, 860-874 (2016).

8. Esterhazy, D. et al. Classical dendritic cells are required for dietary antigenmediated induction of peripheral Treg cells and tolerance. Nat. Immunol. 17, 545-555 (2016)

9. Spahn, T. W. et al. Mesenteric lymph nodes are critical for the induction of highdose oral tolerance in the absence of Peyer's patches. Eur. J. Immunol. 32, 1109-1113 (2002).

10. Spahn, T. W. et al. Induction of oral tolerance to cellular immune responses in the absence of Peyer's patches. Eur. J. Immunol. 31, 1278-1287 (2001).

11. Hadis, $U$. et al. Intestinal tolerance requires gut homing and expansion of FoxP3+ regulatory T cells in the lamina propria. Immunity 34, 237-246 (2011).

12. Worbs, $T$. et al. Oral tolerance originates in the intestinal immune system and relies on antigen carriage by dendritic cells. J. Exp. Med. 203, 519-527 (2006).

13. Kim, K. S. et al. Dietary antigens limit mucosal immunity by inducing regulatory T cells in the small intestine. Science, https://doi.org/10.1126/science.aac5560 (2016).

14. Veenbergen, $\mathrm{S}$. et al. Colonic tolerance develops in the iliac lymph nodes and can be established independent of CD103 dendritic cells. Mucosal Immunol., https:// doi.org/10.1038/mi.2015.118 (2015).

15. Chieppa, M., Rescigno, M., Huang, A. Y. \& Germain, R. N. Dynamic imaging of dendritic cell extension into the small bowel lumen in response to epithelial cell TLR engagement. J. Exp. Med. 203, 2841-2852 (2006).

16. Rescigno, M. et al. Dendritic cells express tight junction proteins and penetrate gut epithelial monolayers to sample bacteria. Nat. Immunol. 2, 361-367 (2001).

17. Shen, L., Weber, C. R., Raleigh, D. R., Yu, D. \& Turner, J. R. Tight junction pore and leak pathways: a dynamic duo. Annu. Rev. Physiol. 73, 283-309 (2011).

18. Jang, M. H. et al. Intestinal villous $M$ cells: an antigen entry site in the mucosal epithelium. Proc. Natl Acad. Sci. USA 101, 6110-6115 (2004).

19. Terahara, K. et al. Comprehensive gene expression profiling of Peyer's patch $M$ cells, villous M-like cells, and intestinal epithelial cells. J. immunol. (Baltimore, Md.: 1950) 180, 7840-7846 (2008).

20. McDole, J. R. et al. Goblet cells deliver luminal antigen to CD103+ dendritic cells in the small intestine. Nature 483, 345-349 (2012).

21. Knoop, K. A., McDonald, K. G., McCrate, S., McDole, J. R. \& Newberry, R. D. Microbial sensing by goblet cells controls immune surveillance of luminal antigens in the colon. Mucosal Immunol. 8, 198-210 (2015).

22. Kulkarni, D. H. \& Newberry, R. D. Intestinal Macromolecular Transport Supporting Adaptive Immunity. Cell. Mol. Gastroenterol. hepatol. 7, 729-737 (2019). https:// doi.org/10.1016/j.jcmgh

23. Rimoldi, M. et al. Monocyte-derived dendritic cells activated by bacteria or by bacteria-stimulated epithelial cells are functionally different. Blood 106, 2818-2826 (2005).

24. Vallon-Eberhard, A., Landsman, L., Yogev, N., Verrier, B. \& Jung, S. Transepithelial pathogen uptake into the small intestinal lamina propria. J. Immunol. (Baltimore, Md.: 1950) 176, 2465-2469 (2006).

25. Niess, J. H. et al. CX3CR1-mediated dendritic cell access to the intestinal lumen and bacterial clearance. Science 307, 254-258 (2005).

26. Mazzini, E., Massimiliano, L., Penna, G. \& Rescigno, M. Oral tolerance can be established via gap junction transfer of fed antigens from CX3CR1(+) macrophages to CD103(+) dendritic cells. Immunity 40, 248-261 (2014).

27. Nagatake, T., Fujita, H., Minato, N. \& Hamazaki, Y. Enteroendocrine cells are specifically marked by cell surface expression of claudin- 4 in mouse small intestine. PLoS One 9, e90638 (2014).

28. Noah, T. K. et al. IL-13-induced Intestinal secretory epithelial cell antigen passages are required for lgE-mediated food-induced anaphylaxis. J. Allergy Clin. Immunol., https://doi.org/10.1016/j.jaci.2019.04.030 (2019).

29. Farache, J. et al. Luminal bacteria recruit CD103+ dendritic cells into the intestinal epithelium to sample bacterial antigens for presentation. Immunity $\mathbf{3 8}$ 581-595 (2013)

30. Kim, K. W. et al. In vivo structure/function and expression analysis of the CX3C chemokine fractalkine. Blood 118, e156-e167 (2011).

31. Hapfelmeier, S. et al. Microbe sampling by mucosal dendritic cells is a discrete, MyD88-independent step in DeltainvG S. Typhimurium colitis. J. Exp. Med. 205 437-450 (2008).

32. Cruickshank, S. M. et al. Rapid dendritic cell mobilization to the large intestinal epithelium is associated with resistance to Trichuris muris infection. J. Immunol. 182, 3055-3062 (2009).

33. Shroyer, N. F. et al. Intestine-specific ablation of mouse atonal homolog 1 (Math1) reveals a role in cellular homeostasis. Gastroenterology 132, 2478-2488 (2007).
34. Yang, Q. Requirement of Math1 for secretory cell lineage commitment in the mouse intestine. Science 294, 2155-2158 (2001).

35. Rose, M. F., Ahmad, K. A., Thaller, C. \& Zoghbi, H. Y. Excitatory neurons of the proprioceptive, interoceptive, and arousal hindbrain networks share a developmental requirement for Math1. Proc. Natl Acad. Sci. USA 106, 22462-22467 (2009).

36. Ben-Arie, N. et al. Functional conservation of atonal and Math1 in the CNS and PNS. Development 127, 1039-1048 (2000).

37. Ireland, H., Houghton, C., Howard, L. \& Winton, D. J. Cellular inheritance of a Creactivated reporter gene to determine Paneth cell longevity in the murine small intestine. Dev. Dyn. 233, 1332-1336 (2005).

38. Troughton, W. D. \& Trier, J. S. Paneth and goblet cell renewal in mouse duodenal crypts. J. Cell Biol. 41, 251-268 (1969).

39. Sefik, E. et al. Individual intestinal symbionts induce a distinct population of RORgamma + regulatory T cells. Science 349, 993-997 (2015).

40. Ohnmacht, C. et al. The microbiota regulates type 2 immunity through RORgammat+ T cells. Science, https://doi.org/10.1126/science.aac4263 (2015).

41. Knoop, K. A. et al. Microbial antigen encounter during a preweaning interval is critical for tolerance to gut bacteria. Sci. Immunol. https://doi.org/10.1126/ sciimmunol.aao1314 (2017).

42. Chai, J. N. et al. Helicobacter species are potent drivers of colonic T cell responses in homeostasis and inflammation. Sci. Immunol. https://doi.org/10.1126/ sciimmunol.aal5068 (2017).

43. $\mathrm{Xu}, \mathrm{M}$. et al. c-MAF-dependent regulatory $\mathrm{T}$ cells mediate immunological tolerance to a gut pathobiont. Nature 554, 373-377 (2018).

44. Ladinsky, M. S. et al. Endocytosis of commensal antigens by intestinal epithelial cells regulates mucosal $T$ cell homeostasis. Science https://doi.org/10.1126/ science.aat4042 (2019).

45. McDonald, K. G. et al. Epithelial expression of the cytosolic retinoid chaperone cellular retinol binding protein II is essential for in vivo imprinting of local gut dendritic cells by lumenal retinoids. Am. J. Pathol. 180, 984-997 (2012).

46. Coombes, J. L. et al. A functionally specialized population of mucosal CD103+ DCs induces Foxp3+ regulatory T cells via a TGF-beta and retinoic aciddependent mechanism. J. Exp. Med. 204, 1757-1764 (2007).

47. Sun, C. M. et al. Small intestine lamina propria dendritic cells promote de novo generation of Foxp3 T reg cells via retinoic acid. J. Exp. Med. 204, 1775-1785 (2007).

48. Mora, J. R. \& von Andrian, U. H. Role of retinoic acid in the imprinting of guthoming IgA-secreting cells. Semin. Immunol. 21, 28-35 (2009).

49. Herzog, R. W. et al. Oral tolerance induction in hemophilia B dogs fed with transplastomic lettuce. Mol. Ther. 25, 512-522 (2017).

50. Chen, $X$. et al. Oral administration of visceral adipose tissue antigens ameliorates metabolic disorders in mice and elevates visceral adipose tissue-resident CD4+ CD25+ Foxp3+ regulatory $\mathrm{T}$ cells. Vaccine, https://doi.org/10.1016/j. vaccine.2017.07.014 (2017).

51. Thota, L. N., Ponnusamy, T., Philip, S., Lu, X. \& Mundkur, L. Immune regulation by oral tolerance induces alternate activation of macrophages and reduces markers of plaque destabilization in Apobtm2Sgy/Ldlrtm1Her/J mice. Sci. Rep. 7, 3997 (2017).

52. Kretschmer, K. et al. Inducing and expanding regulatory $\mathrm{T}$ cell populations by foreign antigen. Nat. Immunol. 6, 1219-1227 (2005).

53. Mucida, D. et al. Oral tolerance in the absence of naturally occurring Tregs. J. Clin. Invest 115, 1923-1933 (2005).

54. Ostroukhova, M. et al. Tolerance induced by inhaled antigen involves CD4(+) T cells expressing membrane-bound TGF-beta and FOXP3. J. Clin. Invest 114 28-38 (2004).

55. Mucida, D. et al. Reciprocal $\mathrm{TH} 17$ and regulatory $\mathrm{T}$ cell differentiation mediated by retinoic acid. Science 317, 256-260 (2007).

56. Mora, J. R. et al. Selective imprinting of gut-homing $T$ cells by Peyer's patch dendritic cells. Nature 424, 88-93 (2003).

57. Mora, J. R. et al. Generation of gut-homing IgA-secreting B cells by intestinal dendritic cells. Science 314, 1157-1160 (2006).

58. Jaensson, E. et al. Small intestinal CD103+ dendritic cells display unique functional properties that are conserved between mice and humans. J. Exp. Med. 205 2139-2149 (2008).

59. Hammerschmidt, S. I. et al. Stromal mesenteric lymph node cells are essential for the generation of gut-homing T cells in vivo. J. Exp. Med. 205, 2483-2490 (2008).

60. Cording, S. et al. The intestinal micro-environment imprints stromal cells to promote efficient Treg induction in gut-draining lymph nodes. Mucosal Immunol. 7, 359-368 (2014).

61. McDonald, K. G. et al. CCR6 promotes steady state intestinal mononuclear phagocyte association with the intestinal epithelium, imprinting, and immune surveillance. Immunology, https://doi.org/10.1111/imm.12801 (2017).

62. Jaensson-Gyllenback, E. et al. Bile retinoids imprint intestinal CD103+ dendritic cells with the ability to generate gut-tropic T cells. Mucosal Immunol. https://doi. org/10.1038/mi.2010.91 (2011). 
Goblet cell associated antigen passages support the induction and... DH. Kulkarni et al.

63. Shan, M. et al. Mucus enhances gut homeostasis and oral tolerance by delivering immunoregulatory signals. Science 342, 447-453 (2013).

64. Atarashi, K. et al. Induction of colonic regulatory $\mathrm{T}$ cells by indigenous Clostridium species. Science 331, 337-341 (2011).

65. Atarashi, K. et al. T induction by a rationally selected mixture of Clostridia strains from the human microbiota. Nature, https://doi.org/10.1038/nature12331 (2013).

66. Round, J. L. \& Mazmanian, S. K. Inducible Foxp3+ regulatory T-cell development by a commensal bacterium of the intestinal microbiota. Proc. Natl Acad. Sci. USA 107, 12204-12209 (2010).

67. Mazmanian, S. K., Round, J. L. \& Kasper, D. L. A microbial symbiosis factor prevents intestinal inflammatory disease. Nature 453, 620-625 (2008).

68. $\mathrm{Chu}, \mathrm{H}$. et al. Gene-microbiota interactions contribute to the pathogenesis of inflammatory bowel disease. Science 352, 1116-1120 (2016).

69. Furusawa, Y. et al. Commensal microbe-derived butyrate induces the differentiation of colonic regulatory T cells. Nature 504, 446-450 (2013).

70. Arpaia, N. et al. Metabolites produced by commensal bacteria promote peripheral regulatory T-cell generation. Nature 504, 451-455 (2013).

71. FOTI, M. \& RICCIARDICASTAGNOLI, P. Antigen sampling by mucosal dendritic cells. Trends Mol. Med. 11, 394-396 (2005).

72. Schulz, O. \& Pabst, O. Antigen sampling in the small intestine. Trends Immunol. 34, 155-161 (2013).

73. Knoop, K. A., Miller, M. J. \& Newberry, R. D. Transepithelial antigen delivery in the small intestine: different paths, different outcomes. Curr. Opin. Gastroenterol. 29, 112-118 (2013).

74. Rescigno, M., Rotta, G., Valzasina, B. \& Ricciardi-Castagnoli, P. Dendritic cells shuttle microbes across gut epithelial monolayers. Immunobiology 204, 572-581 (2001).

75. Morita, N. et al. GPR31-dependent dendrite protrusion of intestinal CX3CR1(+) cells by bacterial metabolites. Nature, https://doi.org/10.1038/s41586-019-0884-1 (2019).

76. Knoop, K. A. et al. RANKL is necessary and sufficient to initiate development of antigen-sampling M cells in the intestinal epithelium. J. Immunol. 183, 5738-5747 (2009).
77. Hase, K. et al. Uptake through glycoprotein 2 of $\mathrm{FimH}(+)$ bacteria by $\mathrm{M}$ cells initiates mucosal immune response. Nature 462, 226-230 (2009).

78. Knoop, K. A., McDonald, K. G., Kulkarni, D. H. \& Newberry, R. D. Antibiotics promote inflammation through the translocation of native commensal colonic bacteria. Gut 65, 1100-U1160 (2016).

79. Knoop, K. A. et al. Antibiotics promote the sampling of luminal antigens and bacteria via colonic goblet cell associated antigen passages. Gut Microbes https:// doi.org/10.1080/19490976.2017.1299846 (2017).

80. Bouziat, R. et al. Reovirus infection triggers inflammatory responses to dietary antigens and development of celiac disease. Science 356, 44-50 (2017).

81. Kulkarni, D. H. et al. Goblet cell associated antigen passages are inhibited during Salmonella typhimurium infection to prevent pathogen dissemination and limit responses to dietary antigens. Mucosal Immunol. https://doi.org/10.1038/s41385018-0007-6 (2018).

82. Barnden, M. J., Allison, J., Heath, W. R. \& Carbone, F. R. Defective TCR expression in transgenic mice constructed using CDNA-based alpha- and beta-chain genes under the control of heterologous regulatory elements. Immunol. Cell Biol. 76, 34-40 (1998).

83. Lindquist, R. L. et al. Visualizing dendritic cell networks in vivo. Nat. Immunol. 5, 1243-1250 (2004).

84. Jung, S. et al. Analysis of fractalkine receptor CX(3)CR1 function by targeted deletion and green fluorescent protein reporter gene insertion. Mol. Cell Biol. 20, 4106-4114 (2000).

85. Kim, J. M., Rasmussen, J. P. \& Rudensky, A. Y. Regulatory T cells prevent catastrophic autoimmunity throughout the lifespan of mice. Nat. Immunol. 8, 191-197 (2007).

86. el Marjou, F. et al. Tissue-specific and inducible Cre-mediated recombination in the gut epithelium. Genesis 39, 186-193 (2004).

87. Lee, T. C. \& Threadgill, D. W. Generation and validation of mice carrying a conditional allele of the epidermal growth factor receptor. Genesis 47, 85-92 (2009).

88. Jeon, J. et al. A subpopulation of neuronal M4 muscarinic acetylcholine receptors plays a critical role in modulating dopamine-dependent behaviors. J. Neurosci. 30, 2396-2405 (2010). 